Published in final edited form as:

Nat Cell Biol. 2013 November ; 15(11): 1340-1350. doi:10.1038/ncb2860.

\title{
Sin1 phosphorylation impairs mTORC2 complex integrity and inhibits downstream Akt signaling to suppress tumorigenesis
}

\author{
Pengda Liu ${ }^{1}$, Wenjian Gan ${ }^{1}$, Hiroyuki Inuzuka ${ }^{1}$, Adam S Lazorchak ${ }^{2}$, Daming Gao ${ }^{1}$, \\ Omotooke Arojo ${ }^{2}$, Dou Liu' ${ }^{2}$, Lixin Wan ${ }^{1}$, Bo Zhai ${ }^{3}$, Yonghao $\mathrm{Yu}^{3,4}$, Min Yuan ${ }^{5}$, Byeong Mo \\ Kim $^{6}$, Shavali Shaik ${ }^{1}$, Suchithra Menon ${ }^{7}$, Steven P. Gygi ${ }^{3}$, Tae Ho Lee ${ }^{6}$, John M Asara ${ }^{5}$, \\ Brendan D. Manning ${ }^{7}$, John Blenis ${ }^{3}$, Bing Su${ }^{2}$, and Wenyi Wei ${ }^{1,8}$ \\ ${ }^{1}$ Department of Pathology, Beth Israel Deaconess Medical Center, Harvard Medical School, \\ Boston, MA 02215 \\ 2Department of Immunobiology and The Vascular Biology and Therapeutics Program, Yale \\ Medical School, New Haven, CT 06520 \\ ${ }^{3}$ Department of Cell Biology, Harvard Medical School, Boston, MA 02115 \\ ${ }^{4}$ Department of Biochemistry, UT Southwestern Medical Center, Dallas, TX 75390 \\ ${ }^{5}$ Department of Medicine, Beth Israel Deaconess Medical Center, Harvard Medical School, \\ Boston, MA 02215 \\ ${ }^{6}$ Division of Gerontology, Department of Medicine, Beth Israel Deaconess Medical Center, \\ Boston, MA 02215 \\ ${ }^{7}$ Department of Genetics \& Complex Diseases, Harvard School of Public Health, Boston, MA \\ 02115
}

\begin{abstract}
The mechanistic target of rapamycin (mTOR) functions as a critical regulator of cellular growth and metabolism by forming multi-component, yet functionally distinct complexes mTORC1 and mTORC2. Although mTORC2 has been implicated in mTORC1 activation, little is known about how mTORC2 is regulated. Here we report that phosphorylation of Sin1 at T86 and T398 suppresses mTORC2 kinase activity by dissociating Sin1 from mTORC2. Importantly, Sin1 phosphorylation, triggered by S6K or Akt, in a cellular context-dependent manner, inhibits not only insulin/IGF-1-mediated, but also PDGF or EGF-induced Akt phosphorylation by mTORC2, demonstrating a negative regulation of mTORC2 independent of IRS-1 and Grb10. Lastly, a
\end{abstract}

\footnotetext{
Users may view, print, copy, download and text and data- mine the content in such documents, for the purposes of academic research, subject always to the full Conditions of use: http://www.nature.com/authors/editorial_policies/license.html\#terms

${ }^{8}$ To whom correspondence should be addressed: Wenyi Wei, Ph.D., Department of Pathology, Beth Israel Deaconess Medical Center, Harvard Medical School, 330 Brookline Ave, Boston, MA 02215, Phone: 617-735-2495, wwei2@ bidmc.harvard.edu.

Author Contributions

P.L., W.G and H.I. performed most of the experiments with assistance from D.G., L.W., A.L., S.S. and O.A. W.W. and B.S. designed the experiments and supervised the study. P.L. and W.W. wrote the manuscript. All authors commented on the manuscript.

Competing Financial Interests

The authors declare no competing financial interests.

Note: Supplementary Information is available in the online version of the paper.
} 
cancer patient-derived Sin1-R81T mutation impairs Sin1 phosphorylation, leading to hypermTORC2 activation via bypassing this negative regulation. Together, our work reveals a Sin 1 phosphorylation-dependent mTORC 2 regulation, providing a potential molecular mechanism by which mutations in the mTORC1/S6K/Sin1 signaling axis might cause aberrant hyper-activation of mTORC2/Akt that facilitates tumorigenesis.

\section{Introduction}

mTOR is a highly conserved important regulator of cell growth and proliferation in a plethora of biological settings in all eukaryotes ${ }^{1-8}$. As such, deregulated mTOR function leads to a variety of human diseases, ranging from cancer ${ }^{5,9}$ to immune dysfunction ${ }^{3}$ and diabetes ${ }^{4,10}$. mTOR functions as a critical and essential catalytic core in at least two known functionally distinct complexes, mTORC1 and mTORC2 ${ }^{3,5}$. A unique subset of associated proteins defines each complex, such as Raptor ${ }^{11}$ in mTORC1, or Rictor ${ }^{12}$ and Sin $1{ }^{13-15}$ in mTORC2. Biologically, mTORC2 mainly promotes cell proliferation and survival 2,16 through phosphorylation of the AGC kinase family members Akt and SGK ${ }^{6}$, in addition to its initially defined role in regulating cell skeletal organization ${ }^{17}$. On the other hand, mTORC1 directly promotes mRNA translation and protein synthesis by phosphorylating S6K1 and 4EBP1 ${ }^{18}$, inhibits autophagy through phosphorylating ULK $1{ }^{19}$ and indirectly enhances ribosome biogenesis via promoting nucleophosmin (NPM) oncogene translation ${ }^{20}$. As most mTORC1 functions are high energy consuming, regulation of mTORC1 activity is tightly coupled to the energy status of the cell and regulated by nutrients, energy, stress and growth factors, thereby ensuring that cells stop growing under unfavorable conditions $1,8,21,22$.

Compared to well-defined mechanisms of mTORC1 activation such as mTORC2/Aktmediated phosphorylation of TSC2 ${ }^{23,24}$ or PRAS40 ${ }^{25,26}$, the upstream signaling that governs mTORC2 activation is just beginning to be appreciated. To this end, mTORC2's association with ribosome was recently found to be necessary for its activation ${ }^{4}$. Furthermore, mTORC1/S6K-mediated phosphorylation of IRS-1 27,28 and Grb10 28,29 also constitutes negative feedback mechanisms to block mTORC 2 activation by insulin/IGF-1. However, both IRS-1 and Grb10 function by suppressing insulin/IGF-1 signaling upstream of PI3K to affect both mTORC1 and mTORC2. Thus, it remains elusive whether mTORC1 could directly regulate mTORC2/Akt without broadly suppressing the PI3K pathway and how mTORC1 suppresses mTORC2/Akt in stimulation conditions other than insulin/IGF-1.

Here we show that in response to a wide spectrum of stimuli including insulin, IGF-1, PDGF and EGF, phosphorylation of Sin1 dissociates Sin 1 from mTORC2 to terminate mTORC2 kinase activity, revealing a negative regulation of mTORC2 function independent of the previously identified negative feedback regulators IRS-1 and Grb10 in suppressing mTORC2. 


\section{Results}

\section{S6K phosphorylates Sin1 on both T86 and T398 sites}

In keeping with previous reports ${ }^{27-30}$, an inverse correlation between mTORC1/S6K and mTORC2 activities was confirmed (Supplementary Fig. S1a-d). Intriguingly, the critical role of Grb10 29,30 and IRS-1 27,28 in regulation of mTORC2/Akt was found to be restricted to insulin/IGF-1, but not PDGF or EGF stimulation (Supplementary Fig. S1e-g). Consistently, inhibition of mTORC1/S6K signaling by a specific S6K1 inhibitor, S6K1-I ${ }^{31}$ or mTORC1 inhibitor, rapamycin ${ }^{11,32,33}$ could still augment Akt activation in TSC2-/MEFs depleted of endogenous IRS-1 and/or Grb10 (Supplementary Fig. S1h-m), advocating that mTORC1/S6K could exert its negative regulation of mTORC2/Akt following PDGF or EGF stimulation through uncharacterized negative feedback loop(s) other than IRS-1 and Grb10. As Rictor and Sin1 are the only two unique essential mTORC2 components ${ }^{13,34}$, we reasoned that the mTORC1/S6K-dependent regulation of mTORC2 might occur through Rictor or Sin1. However, S6K-dependent phosphorylation of Rictor does not affect mTORC2 kinase activity $32,35,36$, which prompted us to investigate whether Sin1 is the primary target to mediate the regulation of mTORC 2 by mTORC1/S6K.

In line with previous reports ${ }^{13,15}$, insulin, IGF-1, PDGF and EGF all could effectively induce Akt S473 phosphorylation in WT but not Sin ${ }^{-/-}$MEFs (Supplementary Fig. S1n) or Sin 1 depleted $T S C 2^{-/}$cells (Supplementary Fig. S1o). This finding prompted us to further examine whether other than IRS-1 and Grb10, modifications of Sin1 may play a critical role in mTORC1-mediated feedback regulation of mTORC2. In support of this hypothesis, phosphorylation of Sin1 was significantly reduced upon inhibition of mTORC1 or S6K, and moderately decreased by the Akt inhibitor, AktVIII (Fig. 1a). Consistently, we detected in vivo Sin1 phosphorylation triggered by S6K1 and to a lesser extent, Akt1, but not other characterized AGC kinases (Fig. 1b and Supplementary Fig. S2a). Notably, S6K1 inhibition led to reduced Sin1 phosphorylation while overexpressing a WT-S6K1 (Fig. 1c), but not a kinase-dead-S6K1 mutant ${ }^{37}$ (Supplementary Fig. S2b), augmented Sin1 phosphorylation. Consistently, depletion of TSC2, which resulted in elevated S6K activity, also led to increased Sin1 phosphorylation (Supplementary Fig. S2c). In support of Sin1 being a S6K substrate, we identified two canonical AGC family kinase-recognition motifs (RxRxxp/ pT) ${ }^{38}$ located at T86 and T398, respectively (Fig. 1d). Notably, mutation of both sites to alanines abolished S6K1-mediated Sin1 phosphorylation in cells (Fig. 1e) or in vitro (Fig. 1f). Interestingly, mutation of either T86 or T398 to an alanine did not completely abolish the S6K-dependent phosphorylation of Sin1 (Fig. 1e), indicating that both T86 and T398 might be potential S6K1 sites in vivo.

Importantly, phosphorylation of T86 and T398 were detected by mass spectrometry (Supplementary Fig. S2d-e). To gain further mechanistic insights into how Sin1 phosphorylation may affect mTORC2 activation under physiological conditions, we developed phospho-specific antibodies against pT86-Sin1 or pT398-Sin1, respectively (Supplementary Fig. S2f-g). Using these antibodies we observed an increase in Sin1-pT86 and Sin1-pT398 upon induction by insulin (Fig. 1g and Supplementary Fig. S2h), IGF-1, PDGF or EGF (Fig. 1h), whereas these phosphorylation events could be attenuated by 
inactivating S6K1 either through depleting Raptor (Fig. 1h and Supplementary Fig. S2i), or by S6K1-I (Supplementary Fig. S2j). Notably, S6K1 knockdown only partially reduced Sin1 phosphorylation (Supplementary Fig. S2i), suggesting that other S6K isoforms might phosphorylate Sin1. Nevertheless, recombinant S6K1 phosphorylated Sin1 in vitro (Supplementary Fig. S2k) and conversely, rapamycin could attenuate Sin1-pT86 mediated by WT-S6K1 but not a rapamycin-resistant form of S6K1 ${ }^{37}$ (Supplementary Fig. 21), further supporting S6K1 as a physiological kinase for Sin1.

\section{Both Akt and S6K may phosphorylate Sin1 in a context-dependent manner}

Although depletion of endogenous Akt1 also led to a moderate decrease in Sin1 phosphorylation, its effects were less compared to inhibiting S6K1 or mTORC1 in our experimental conditions (Supplementary Fig. S2m-n). Moreover, Akt might regulate Sin1 phosphorylation indirectly through activating mTORC1/S6K ${ }^{11,13}$. In support of this model, Sin1-T86 phosphorylation correlated positively with S6K1 activity (evidenced by pS6) but inversely with Akt phosphorylation (Supplementary Fig. S2o-p), indicating that Sin1 phosphorylation by S6K1 might act as a physiological negative regulator of Akt phosphorylation in fibroblasts. However, this conclusion might be cellular contextdependent as a recent study has suggested Akt as the Sin1-T86 phosphorylating kinase in adipocytes 39 .

To gain further mechanistic insights into upstream kinases responsible for phosphorylating Sin1, we examined the possible role of S6K versus Akt in mediating Sin1 phosphorylation on T86 and T398 in 3T3-L1 cells ${ }^{40}$. Consistent with Humphrey et al ${ }^{39}$, Sin1-pT86 signals were largely blocked by Akt inhibition, but only moderately reduced by inhibiting mTORC1 or S6K (Supplementary Fig. S2q), confirming Akt as the major Sin1-T86 phosphorylating kinase in 3T3-L1 cells. However, inhibitors targeting either Akt or S6K1 led to a dramatic reduction in Sin1-pT398, suggesting that both Akt and S6K are involved in phosphorylating Sin1-T398 in 3T3-L1 adipocytes. On the other hand, in epithelium-derived HeLa cells, in stark contrast to 3T3-L1, inhibition of mTORC1/S6K, rather than Akt, led to a more severe reduction in both Sin1-pT86 and Sin1-pT398 signals (Supplementary Fig S2r), indicating that in HeLa cells, S6K, but not Akt, is the major kinase responsible for Sin1-T86 and -T398 phosphorylation. Collectively, these results implicate that physiological upstream kinase(s) responsible for Sin1-T86 or Sin1-T398 phosphorylation might be tissue-specific or cellular context-dependent. A similar tissue specific phosphorylation atlas of mouse proteins has been established ${ }^{41}$. Hence, adipocytes versus epithelial cells might require tightly regulated and specialized, phosphorylation-dependent intracellular signaling, thereby utilizing distinct upstream signaling routes to regulate effector pathways such as mTORC2. As carcinomas primarily derive from epithelial cells ${ }^{42,43}$, we chose to focus on understanding how regulation of mTORC 2 by S6K-mediated Sin1 phosphorylation contributes to tumorigenesis in the epithelial cell settings.

\section{Phosphorylation of Sin1 at both T86 and T398 dissociates Sin1 from the mTORC2 complex}

As Sin1 is an essential component that governs mTORC2 integrity ${ }^{13-15}$, we next examined whether Sin1 phosphorylation on T86 and T398 affects mTORC2 function. Strikingly, compared to WT-Sin1, the Sin1 phospho-mimetic mutant (T86E/T398E, Sin1-EE), failed to 
interact with the essential mTORC2 components Rictor, mTOR or G $\beta$ L (Fig. 2a and Supplementary Fig. S3a-c), indicating that the assembly of a functional mTORC2 complex was prevented by Sin 1 phosphorylation. In supporting of this model, S6K1-mediated phosphorylation of Sin1-WT, but not the Sin1 phospho-deficient mutant (T86A/T398A, Sin1-AA), reduced Sin1 interaction with Rictor (Fig. 2b), mTOR (Fig. 2c) and GßL (Fig. 2d). Notably, disruption of the Sin1/Rictor interaction required Sin1 phosphorylation on both T86 and T398 (Supplementary Fig. S3d,e). To further investigate the underlying molecular mechanism(s), we performed gel-filtration assays to examine mTORC2 complex assembly in cells, and observed that WT-Sin1, but not Sin1-EE, co-eluted with mTORC2 components including Rictor, mTOR, and G $\beta \mathrm{L}$ (Fig. 2e). Furthermore, depletion of TSC2 led to elevated mTORC1/S6K activity and shifted a significant portion of Sin1 to mTORC2free fractions (Supplementary Fig. S3f,g). Strikingly, the mTORC2-free Sin1 species were enriched with pT86-Sin1 (Supplementary Fig. S3f). Consistently, less Rictor was detected in association with Sin1 in $T S C 2^{-/-}$cells (Fig. 2f), whereas inhibiting S6K1 activity in $T S C 2^{-/-}$cells by S6K1-I partially restored Rictor interaction with Sin1 (Supplementary Fig. S3h,i), supporting a crucial role of S6K in regulating the Sin1-Rictor interaction.

To further understand the role of each Sin 1 phosphorylation event in mediating mTORC2 complex organization, we truncated Sin1 into four regions (Fig. $2 \mathrm{~g}$ ) and observed that Sin1 mainly interacts with Rictor through its $\mathrm{N}$-terminal region, with mTOR kinase domain via its PH domain, and with GßL through its N, RBD and PH domains (Fig. 2h). Interestingly, phosphorylation of Sin1 at T86 in the N-terminal region abolished Sin1-N interaction with Rictor (Fig. 2i), while phosphorylation on T398 in the PH domain reduced Sin1 interaction with the mTOR kinase domain (Fig. 2j), indicating the distinct role of each phosphorylation site in possibly mediating the organization of the mTORC 2 complex, further supporting that phosphorylation of both T86 and T398 is required to functionally inactivate mTORC2.

More importantly, under physiological stimulation conditions such as EGF (Fig. 3a and Supplementary Fig. S4a) or insulin (Fig. 3b and Supplementary Fig. S4b) induction, we observed an inverse correlation between the induced Sin1 phosphorylation and a dissociation of Sin 1 from Rictor or mTOR, which could be abolished by mutating either T86 or T398 to alanine (Fig. 3c), or by rapamycin treatment (Supplementary Fig. S4c-f), demonstrating a physiological role of Sin 1 phosphorylation in negatively regulating mTORC2 function. Notably, compared with Sin1-WT, impairing Sin1 phosphorylation by mutating either T86 or T398 (Fig. 3d), inhibiting S6K activity (Fig. 3e and Supplementary Fig. S4g), or depleting endogenous Raptor (Supplementary Fig. S4h,i), led to sustained Akt phosphorylation under EGF or insulin stimulation. Conversely, activating S6K by either depletion of endogenous TSC2 (Supplementary Fig. S4j) or genetic ablation of TSC2 (Supplementary Fig. S4k), resulted in reduced Akt S473 phosphorylation. In keeping with previous reports ${ }^{27,44}$, Akt phosphorylation in response to various stimuli was attenuated in $\mathrm{TSC}^{-/-}$cells with elevated S6K activity (Supplementary Fig. S41), confirming a negative role for S6K-mediated phosphorylation of Sin1 in Akt activation. 


\section{Phosphorylation of Sin1 on both T86 and T398 suppresses mTORC2-mediated activation of Akt}

Notably, Sin1 phosphorylation did not affect Sin1 stability (Supplementary Fig. S4m,n). However, phosphorylation of Sin1 specifically reduced its interaction with the mTORC2 substrate, Akt1 (Fig. 4a-c) but not with another characterized mTORC2 substrate SGK1 ${ }^{45,46}$ (Fig. 4d-e), nor the other AGC kinase S6K1 (Fig. 4f-g), which might explain why Sin1 phosphorylation leads to impaired Akt activation.

To further verify the critical roles of Sin1 phosphorylation in regulating mTORC2 kinase activity, we performed in vitro kinase assays to show that unlike Sin1-WT or Sin1-AA, immunoprecipitated Sin1-EE failed to phosphorylate GST-Akt1 in vitro, presumably due to its deficiency in forming a functional mTORC2 complex (Fig. 5a-b). By coupling gel filtration with in vitro kinase assays, we demonstrated that only the gel-filtration fractions with molecular weights corresponding to the full mTORC2 complex retained the mTORC2 kinase activity towards GST-Akt in vitro (Fig. 5c-d). Strikingly, consistent with previous studies ${ }^{47-49}$, insulin stimulation led to an oscillated pattern of Akt activation with reduced Akt phosphorylation after prolonged treatment in various cell lines (Supplementary Fig. S5a-c), a phenotype observed in many signaling pathways ${ }^{47,50}$. However, the underlying mechanism of the oscillation of Akt phosphorylation remains not fully understood. Notably, prolonged (45 minutes of) insulin treatment led to a severe reduction of mTORC2-mediated in vitro phosphorylation of Akt in part by inducing pT86-Sin1 (Fig. 5c-d) to disrupt the mTORC2 complex integrity. More importantly, rapamycin treatment effectively restored mTORC2 complex integrity and kinase activity under prolonged EGF stimulation (Fig. 5e), emphasizing that $\mathrm{S} 6 \mathrm{~K}$ negatively regulates mTORC2 activity in part through phosphorylating Sin1, serving as a molecular switch to shut down EGF or insulin-induced activation of mTORC2/Akt in a timely fashion (Supplementary Fig. S5d).

Given the critical role of the mTORC2/Akt signaling in cell survival and tumorigenesis, we next examined the biological significance of S6K-mediated Sin1 phosphorylation on mTORC2 function. Consistent with in vitro kinase assay results (Fig. 5a), Sin1-WT, but not Sin1-EE, rescued in vivo Akt-S473 phosphorylation in Sin1 ${ }^{-/-}$MEFs (Fig. 6a,b and Supplementary Fig. S6a) ${ }^{13,15}$. Furthermore, in echoing the in vitro kinase results (Fig. 6c), immuno-purified endogenous Akt1 from Sin1-EE-expressing Sin1 $1^{-/-}$MEFs was deficient in phosphorylating the well-characterized Akt substrate, crosstide ${ }^{51,52}$ in vitro (Fig. 6d). Consistently, compared with Sin1-WT expressing $\operatorname{Sin} 1^{-/-}$cells, Sin $1^{-/-}$cells expressing Sin1-EE exhibited reduced levels of phospho-FOXOs (Fig. 6a) ${ }^{53}$, thereby more sensitive to cell death ${ }^{54,55}$ induced by etoposide (Fig. 6e,f and Supplementary Fig. S6b-e) or cisplatin (Fig. 6g). Similar apoptotic sensitization was observed after introducing Sin1-EE, in comparison to Sin1-WT, into Sin1-depleted OVCAR5 cells (Supplementary Fig. S6f-i, supporting a physiological role of Sin1 phosphorylation in regulating cellular survival in part through the Akt/FOXO signaling pathway 56,57 . 


\section{The ovarian cancer patient-derived Sin1-R81T mutation displayed elevated oncogenic activity in part by bypassing Sin1 phosphorylation-mediated suppression of mTORC2/Akt signaling}

Interestingly, two natural Sin1 mutations (R81T and S84L) were recently identified in ovarian cancer patients 58 and skin cancer patients, respectively (Fig. 7a) from two publically available cancer genome databases: the Cosmic database (http:// cancer.sanger.ac.uk/cancergenome/projects/cosmic/) ${ }^{59}$ and the CBio database (http:// www.cbioportal.org/public-portal/) ${ }^{60}$. Notably, these mutations appear to disrupt the canonical S6K phosphorylation motif (Fig. 7a), and indeed we observed a significant reduction in pT86 in both Sin1-R81T (Fig. 7b) and S84L (Supplementary Fig. S7a-b) mutants, suggesting that they functionally mimicked Sin1-T86A. More importantly, a synthesized pT86-containing peptide could be efficiently recognized by the Sin1-pT86 antibody regardless of Sin1-R81T mutation status (Fig. 7c), excluding a secondary antibody effect. As the R81T mutant impairs the stringent AGC substrate motif, we focused on studying the pathophysiological effects of this mutant in cells.

We found that Sin1-pT86 signal was nearly abolished in the Sin1-R81T mutant in response to a wide spectrum of stimuli, including insulin, IGF-1, PDGF and EGF (Fig. 7d). Unlike WT-Sin1 (Fig. 3b-c), R81T-Sin1 demonstrated a relatively sustained interaction with mTORC2 components (Fig. 7e and Supplementary Fig. S7c,d), resulting in prolonged mTORC2 kinase activity towards phosphorylating Akt in vitro (Fig. 5d). In keeping with these results, in response to various external stimuli including EGF (Fig. 7f and Supplementary Fig. S7e), insulin (Fig. 7g) and PDGF (Supplementary Fig. S7f), there is a more sustained Akt activation in Sin1-R81T compared with Sin1-WT expressing OVCAR5 cells. These results support a model that the R81T mutation may enhance tumorigenesis in part through augmenting Akt activation (Supplementary Fig. S7g).

Biologically, expression of Sin1-R81T in either Sin1 $1^{-/-}$MEFs or shSin1-OVCAR5 cells led to elevated phosphorylation of Akt and the Akt substrate FOXOs (Supplementary Fig. S8a,b), which subsequently conferred resistance to etoposide (Fig. 8a and Supplementary Fig. S8c-g) or cisplatin (Fig. 8b,c). Moreover, re-introduction of R81T, but not WT-Sin1, significantly promoted the anchorage-independent growth of Sin1-depleted OVCAR5 cells, arguing that $\mathrm{R} 81 \mathrm{~T}$ is a gain-of-function oncogenic mutation that favors cellular transformation (Fig. 8d). This finding was echoed by the significantly enhanced in vivo tumor formation ability of R81T expressing OVCAR5 cells in a mouse xenograft model, (Fig. 8e-g and Supplementary Fig. 8h). Although further investigation is required, these results provide an important clinical relevance for the oncogenic role of Sin1-R81T mutant, in part by bypassing the mTORC1-S6K-mediated negative feedback regulation on mTORC2/Akt activation. Interestingly, we observed that Sin1-R81T-expressing OVCAR5 cells are more resistant to the Akt inhibitor, AktVIII (Supplementary Fig. S8i-k), indicating that cancer patients bearing the Sin1-R81T mutation might poorly respond to Akt or mTOR inhibitor treatments.

Given that abnormal mTORC2/Akt activities are associated with numerous human diseases ${ }^{3,6,7}$, impaired Sin 1 phosphorylation (such as caused by the R81T mutation) might 
contribute to deregulated mTORC2/Akt activity in many types of tissues and diseases. In this regard, we found that rapamycin treatment attenuated Sin1 T86 phosphorylation in splenic B cells freshly isolated from mice, leading to elevated Akt phosphorylation in vivo (Supplementary Fig. S81-o). Similar results were also observed from rapamycin-treated mouse livers (Fig. 8h). Consistently, an inverse correlation between pT86-Sin1 and pS473Akt was observed in a panel of T-ALL cell lines as well as in ovarian caner patient samples (Fig. 8i and Supplementary Fig. S8p-r), emphasizing the possible pathological importance of the identified negative feedback mechanism in human cancer settings. However, further studies with larger sample sizes are warranted to determine a significant inverse correlation between pT86-Sin1 and pS473-Akt.

\section{Discussion}

Recent studies have begun to reveal a complicated cross-communication between the two mTOR-containing complexes mTORC1 and mTORC2, while the exact molecular mechanism(s) remain largely elusive. Here we define an independent negative feedback regulation through which S6K or Akt directly phosphorylates Sin1 to repress mTORC2 activation in epithelial cells or adipocytes, respectively, thereby providing further molecular insights into a direct and efficient strategy to timely suppress the mTORC2/Akt signaling in a possible tissue and cellular context dependent manner. Nonetheless, phosphorylation of both T86 and T398 of Sin1 is required for the complete inactivation of mTORC2 kinase activity in part by dissociating Sin 1 from other mTORC2 components.

As illustrated in Supplementary Fig. S8s, mTORC1 could be regulated indirectly by mTORC2 in response to extra cellular growth factors, or controlled by multiple in vivo signal fluxes, such as amino acids, nutrients and stresses. As frequently observed in many signaling pathways like those initiated by Ras or PI3K ${ }^{61}$, the mTORC2-mediated growth factor signal is only activated transiently followed by a quench of the signaling through multiple negative feedback regulations to ensure that their activations are presented only in a "pulse" manner ${ }^{62}$. Most significantly, an ovarian cancer patient-derived R81T mutation of Sin 1 could bypass the identified Sin 1 phosphorylation-mediated negative regulation of mTORC2 due to the lack of Sin 1 phosphorylation motif, providing a molecular mechanism for the elevated mTORC2/Akt activation that could potentially promote tumorigenesis in the ovarian cancer settings. In summary, our work identified a negative regulation mechanism mediated largely by Sin 1 phosphorylation on both T86 and T398 to specifically suppress mTORC2 integrity and kinase activity towards Akt-S473 phosphorylation. Moreover, we demonstrated that deregulation of this negative regulation in pathological conditions may contribute to accelerated tumor formation.

\section{Methods}

Methods and any associated references are available in the online version of the paper.

\section{Supplementary Material}

Refer to Web version on PubMed Central for supplementary material. 


\section{Acknowledgments}

We thank Alex Toker, Jianping Guo, Kai Xu, Alan W Lau and Adriana Tron for critical reading of the manuscript, Steve Elledge, Shunsuke Ishii, William Hahn, Dos Sarvassov and Jamie Dempsey for providing valuable reagents, Bruce Spiegelman for providing 3T3-L1 adipocyte cells, Lewis Cantley and Alex Toker for helpful suggestions, and members of the Wei, Blennis and Su laboratories for useful discussions. W.W. is an ACS research scholar and a LLS research scholar. Y. Yu is a CPRIT Scholar (CPRIT R1103) in Cancer Research and a Virginia Murchison Linthicum Scholar in Medical Research. P.L. is NRSA T32 trainee and supported by 5T32HL007893. This work was supported in part by the NIH grants (W.W., GM089763, GM094777 and CA177910; and B.S., AI063348 and PR093728).

\section{References}

1. Zoncu R, Efeyan A, Sabatini DM. mTOR: from growth signal integration to cancer, diabetes and ageing. Nat Rev Mol Cell Biol. 2011; 12:21-35. [PubMed: 21157483]

2. Wullschleger S, Loewith R, Hall MN. TOR signaling in growth and metabolism. Cell. 2006; 124:471-484. [PubMed: 16469695]

3. Laplante M, Sabatini DM. mTOR signaling in growth control and disease. Cell. 2012; 149:274-293. [PubMed: 22500797]

4. Guertin DA, Sabatini DM. Defining the role of mTOR in cancer. Cancer Cell. 2007; 12:9-22. [PubMed: 17613433]

5. Sabatini DM. mTOR and cancer: insights into a complex relationship. Nat Rev Cancer. 2006; 6:729-734. [PubMed: 16915295]

6. Dazert E, Hall MN. mTOR signaling in disease. Curr Opin Cell Biol. 2011; 23:744-755. [PubMed: 21963299]

7. Alayev A, Holz MK. mTOR signaling for biological control and cancer. J Cell Physiol. 2013; 228:1658-1664. [PubMed: 23460185]

8. Jewell JL, Russell RC, Guan KL. Amino acid signalling upstream of mTOR. Nat Rev Mol Cell Biol. 2013; 14:133-139. [PubMed: 23361334]

9. Guertin DA, Sabatini DM. An expanding role for mTOR in cancer. Trends Mol Med. 2005; 11:353361. [PubMed: 16002336]

10. Inoki K, Corradetti MN, Guan KL. Dysregulation of the TSC-mTOR pathway in human disease. Nat Genet. 2005; 37:19-24. [PubMed: 15624019]

11. Kim DH, et al. mTOR interacts with raptor to form a nutrient-sensitive complex that signals to the cell growth machinery. Cell. 2002; 110:163-175. [PubMed: 12150925]

12. Sarbassov DD, et al. Rictor, a novel binding partner of $\mathrm{mTOR}$, defines a rapamycin-insensitive and raptor-independent pathway that regulates the cytoskeleton. Curr Biol. 2004; 14:1296-1302. [PubMed: 15268862]

13. Jacinto E, et al. SIN1/MIP1 maintains rictor-mTOR complex integrity and regulates Akt phosphorylation and substrate specificity. Cell. 2006; 127:125-137. [PubMed: 16962653]

14. Frias MA, et al. mSin1 is necessary for Akt/PKB phosphorylation, and its isoforms define three distinct mTORC2s. Curr Biol. 2006; 16:1865-1870. [PubMed: 16919458]

15. Yang Q, Inoki K, Ikenoue T, Guan KL. Identification of Sin 1 as an essential TORC2 component required for complex formation and kinase activity. Genes Dev. 2006; 20:2820-2832. [PubMed: 17043309]

16. Hung CM, Garcia-Haro L, Sparks CA, Guertin DA. mTOR-dependent cell survival mechanisms. Cold Spring Harb Perspect Biol. 2012; 4

17. Jacinto E, et al. Mammalian TOR complex 2 controls the actin cytoskeleton and is rapamycin insensitive. Nat Cell Biol. 2004; 6:1122-1128. [PubMed: 15467718]

18. Ma XM, Blenis J. Molecular mechanisms of mTOR-mediated translational control. Nat Rev Mol Cell Biol. 2009; 10:307-318. [PubMed: 19339977]

19. Chan EY. mTORC1 phosphorylates the ULK1-mAtg13-FIP200 autophagy regulatory complex. Sci Signal. 2009; 2:pe51. [PubMed: 19690328] 
20. Pelletier CL, et al. TSC1 sets the rate of ribosome export and protein synthesis through nucleophosmin translation. Cancer Res. 2007; 67:1609-1617. [PubMed: 17308101]

21. Dibble CC, Manning BD. Signal integration by mTORC1 coordinates nutrient input with biosynthetic output. Nat Cell Biol. 2013; 15:555-564. [PubMed: 23728461]

22. Efeyan A, Zoncu R, Sabatini DM. Amino acids and mTORC1: from lysosomes to disease. Trends Mol Med. 2012; 18:524-533. [PubMed: 22749019]

23. Inoki K, Li Y, Zhu T, Wu J, Guan KL. TSC2 is phosphorylated and inhibited by Akt and suppresses mTOR signalling. Nat Cell Biol. 2002; 4:648-657. [PubMed: 12172553]

24. Manning BD, Tee AR, Logsdon MN, Blenis J, Cantley LC. Identification of the tuberous sclerosis complex-2 tumor suppressor gene product tuberin as a target of the phosphoinositide 3-kinase/akt pathway. Mol Cell. 2002; 10:151-162. [PubMed: 12150915]

25. Sancak Y, et al. PRAS40 is an insulin-regulated inhibitor of the mTORC1 protein kinase. Mol Cell. 2007; 25:903-915. [PubMed: 17386266]

26. Vander Haar E, Lee SI, Bandhakavi S, Griffin TJ, Kim DH. Insulin signalling to mTOR mediated by the Akt/PKB substrate PRAS40. Nat Cell Biol. 2007; 9:316-323. [PubMed: 17277771]

27. Harrington LS, et al. The TSC1-2 tumor suppressor controls insulin-PI3K signaling via regulation of IRS proteins. J Cell Biol. 2004; 166:213-223. [PubMed: 15249583]

28. Shah OJ, Wang Z, Hunter T. Inappropriate activation of the TSC/Rheb/mTOR/S6K cassette induces IRS1/2 depletion, insulin resistance, and cell survival deficiencies. Curr Biol. 2004; 14:1650-1656. [PubMed: 15380067]

29. Hsu PP, et al. The mTOR-regulated phosphoproteome reveals a mechanism of mTORC1-mediated inhibition of growth factor signaling. Science. 2011; 332:1317-1322. [PubMed: 21659604]

30. Yu Y, et al. Phosphoproteomic analysis identifies Grb10 as an mTORC1 substrate that negatively regulates insulin signaling. Science. 2011; 332:1322-1326. [PubMed: 21659605]

31. Pearce LR, et al. Characterization of PF-4708671, a novel and highly specific inhibitor of p70 ribosomal S6 kinase (S6K1). The Biochemical journal. 2010; 431:245-255. [PubMed: 20704563]

32. Gao D, et al. Rictor forms a complex with Cullin-1 to promote SGK1 ubiquitination and destruction. Mol Cell. 2010; 39:797-808. [PubMed: 20832730]

33. Kim DH, et al. GbetaL, a positive regulator of the rapamycin-sensitive pathway required for the nutrient-sensitive interaction between raptor and mTOR. Mol Cell. 2003; 11:895-904. [PubMed: 12718876]

34. Guertin DA, et al. Ablation in mice of the mTORC components raptor, rictor, or mLST8 reveals that mTORC2 is required for signaling to Akt-FOXO and PKCalpha, but not S6K1. Dev Cell. 2006; 11:859-871. [PubMed: 17141160]

35. Dibble CC, Asara JM, Manning BD. Characterization of Rictor phosphorylation sites reveals direct regulation of mTOR complex 2 by S6K1. Mol Cell Biol. 2009; 29:5657-5670. [PubMed: 19720745]

36. Treins C, Warne PH, Magnuson MA, Pende M, Downward J. Rictor is a novel target of p70 S6 kinase-1. Oncogene. 2010; 29:1003-1016. [PubMed: 19935711]

37. Romanelli A, Dreisbach VC, Blenis J. Characterization of phosphatidylinositol 3-kinase-dependent phosphorylation of the hydrophobic motif site $\operatorname{Thr}(389)$ in p70 S6 kinase 1. J Biol Chem. 2002; 277:40281-40289. [PubMed: 12183455]

38. Obata T, et al. Peptide and protein library screening defines optimal substrate motifs for AKT/ PKB. J Biol Chem. 2000; 275:36108-36115. [PubMed: 10945990]

39. Humphrey SJ, et al. Dynamic Adipocyte Phosphoproteome Reveals that Akt Directly Regulates mTORC2. Cell Metab. 2013; 17:1009-1020. [PubMed: 23684622]

40. Gupta RK, et al. Transcriptional control of preadipocyte determination by Zfp423. Nature. 2010; 464:619-623. [PubMed: 20200519]

41. Huttlin EL, et al. A tissue-specific atlas of mouse protein phosphorylation and expression. Cell. 2010; 143:1174-1189. [PubMed: 21183079]

42. Hanahan D, Weinberg RA. Hallmarks of cancer: the next generation. Cell. 2011; 144:646-674. [PubMed: 21376230]

43. Hanahan D, Weinberg RA. The hallmarks of cancer. Cell. 2000; 100:57-70. [PubMed: 10647931] 
44. Zhang H, et al. PDGFRs are critical for PI3K/Akt activation and negatively regulated by mTOR. J Clin Invest. 2007; 117:730-738. [PubMed: 17290308]

45. Garcia-Martinez JM, Alessi DR. mTOR complex 2 (mTORC2) controls hydrophobic motif phosphorylation and activation of serum- and glucocorticoid-induced protein kinase 1 (SGK1). The Biochemical journal. 2008; 416:375-385. [PubMed: 18925875]

46. Yan L, Mieulet V, Lamb RF. mTORC2 is the hydrophobic motif kinase for SGK1. The Biochemical journal. 2008; 416:e19-21. [PubMed: 19025518]

47. Purvis JE, Lahav G. Encoding and Decoding Cellular Information through Signaling Dynamics. Cell. 2013; 152:945-956. [PubMed: 23452846]

48. Ikeda F, Lahav G. Signal transduction and signaling networks. Mol Biol Cell. 2013; 24:676. [PubMed: 23486400]

49. Purvis JE, Lahav G. Decoding the insulin signal. Mol Cell. 2012; 46:715-716. [PubMed: 22749395]

50. Purvis JE, et al. p53 dynamics control cell fate. Science. 2012; 336:1440-1444. [PubMed: 22700930]

51. Meier R, Alessi DR, Cron P, Andjelkovic M, Hemmings BA. Mitogenic activation, phosphorylation, and nuclear translocation of protein kinase Bbeta. J Biol Chem. 1997; 272:30491-30497. [PubMed: 9374542]

52. Baer K, et al. Activation of a GST-tagged AKT2/PKBbeta. Biochim Biophys Acta. 2005; 1725:340-347. [PubMed: 15890450]

53. Zhang X, Tang N, Hadden TJ, Rishi AK. Akt, FoxO and regulation of apoptosis. Biochim Biophys Acta. 2011; 1813:1978-1986. [PubMed: 21440011]

54. Paik JH, et al. FoxOs are lineage-restricted redundant tumor suppressors and regulate endothelial cell homeostasis. Cell. 2007; 128:309-323. [PubMed: 17254969]

55. Gan B, et al. FoxOs enforce a progression checkpoint to constrain mTORC1-activated renal tumorigenesis. Cancer Cell. 2010; 18:472-484. [PubMed: 21075312]

56. Vogt PK, Jiang H, Aoki M. Triple layer control: phosphorylation, acetylation and ubiquitination of FOXO proteins. Cell Cycle. 2005; 4:908-913. [PubMed: 15917664]

57. Brunet A, et al. Akt promotes cell survival by phosphorylating and inhibiting a Forkhead transcription factor. Cell. 1999; 96:857-868. [PubMed: 10102273]

58. Network CGAR. Integrated genomic analyses of ovarian carcinoma. Nature. 2011; 474:609-615. [PubMed: 21720365]

59. Forbes SA, et al. COSMIC (the Catalogue of Somatic Mutations in Cancer): a resource to investigate acquired mutations in human cancer. Nucleic acids research. 2010; 38:D652-657. [PubMed: 19906727]

60. Cerami E, et al. The cBio cancer genomics portal: an open platform for exploring multidimensional cancer genomics data. Cancer discovery. 2012; 2:401-404. [PubMed: 22588877]

61. Shankaran H, et al. Rapid and sustained nuclear-cytoplasmic ERK oscillations induced by epidermal growth factor. Mol Syst Biol. 2009; 5:332. [PubMed: 19953086]

62. Chen JY, Lin JR, Cimprich KA, Meyer T. A Two-Dimensional ERK-AKT Signaling Code for an NGF-Triggered Cell-Fate Decision. Mol Cell. 2012; 45:196-209. [PubMed: 22206868] 

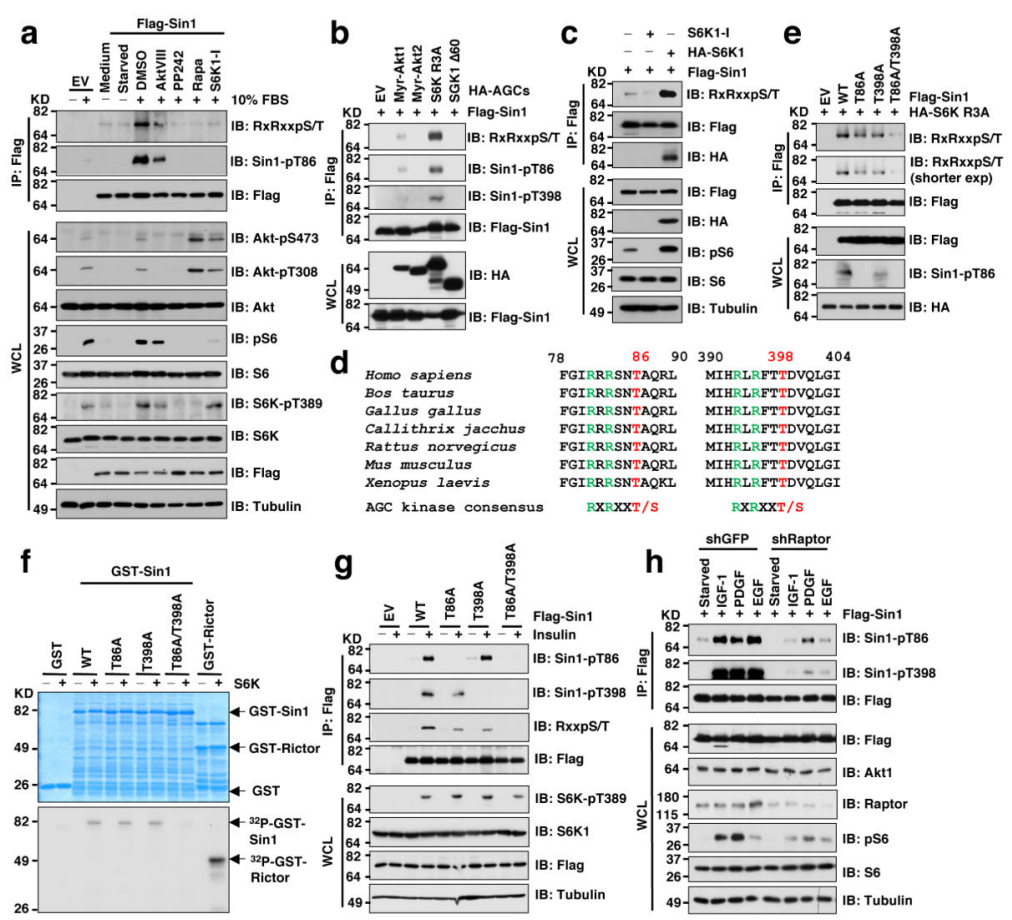

Figure 1. S6K phosphorylates Sin1 on both T86 and T398 sites

a. Immunoblot (IB) analysis of whole cell lysates (WCL) and Flag-immunoprecipitates (IP) derived from Flag-Sin1-transfected HeLa cells that were serum-starved for 24 hours and then collected after serum stimulation for 30 minutes. Where indicated, the kinase inhibitors (AktVIII: $10 \mu \mathrm{M}$, PP242: $1 \mu \mathrm{M}$, Rapamycin: $20 \mathrm{nM}$, S6K1-I: $10 \mu \mathrm{M}$ ) were added together with insulin (100 nM). DMSO was used as a negative control.

b. IB analysis of WCL and IP derived from 293T cells transfected with Flag-Sin1 and the indicated HA-tagged constitutive active AGC family kinases.

c. IB analysis of WCL and IP derived from 293T cells transfected with Flag-Sin1 and HAS6K1 (or empty vector as a negative control). Where indicated, the S6K inhibitor was added.

d. Schematic illustration of the two evolutionarily conserved putative S6K phosphorylation sites, T86 and T398 within Sin1.

e. IB analysis of WCL and IP derived from 293T cells transfected with constitutive active form of S6K (HA-S6K-R3A) and the indicated Flag-Sin1 constructs.

f. In vitro kinase assays depicting major S6K phosphorylation sites in Sin1. Please note that the GST-Rictor fusion protein used here is not the full-length protein but rather the truncated version that contains the S6K phosphorylation site T1135 (GST-Rictor-C-tail [aa13901708]).

g. IB analysis of WCL and Flag-IP derived from HeLa cells transfected with the indicated Flag-Sin 1 constructs. Where indicated, cells were serum starved for 12 hours and stimulated by $100 \mathrm{nM}$ insulin for 30 minutes before harvesting.

h. IB analysis of WCL and Flag-IP derived from HeLa cells depleted of Raptor transfected with Flag-Sin1 (shGFP as a negative control). Where indicated, cells were serum starved for 12 hours and stimulated by the indicated stimuli before harvesting. 


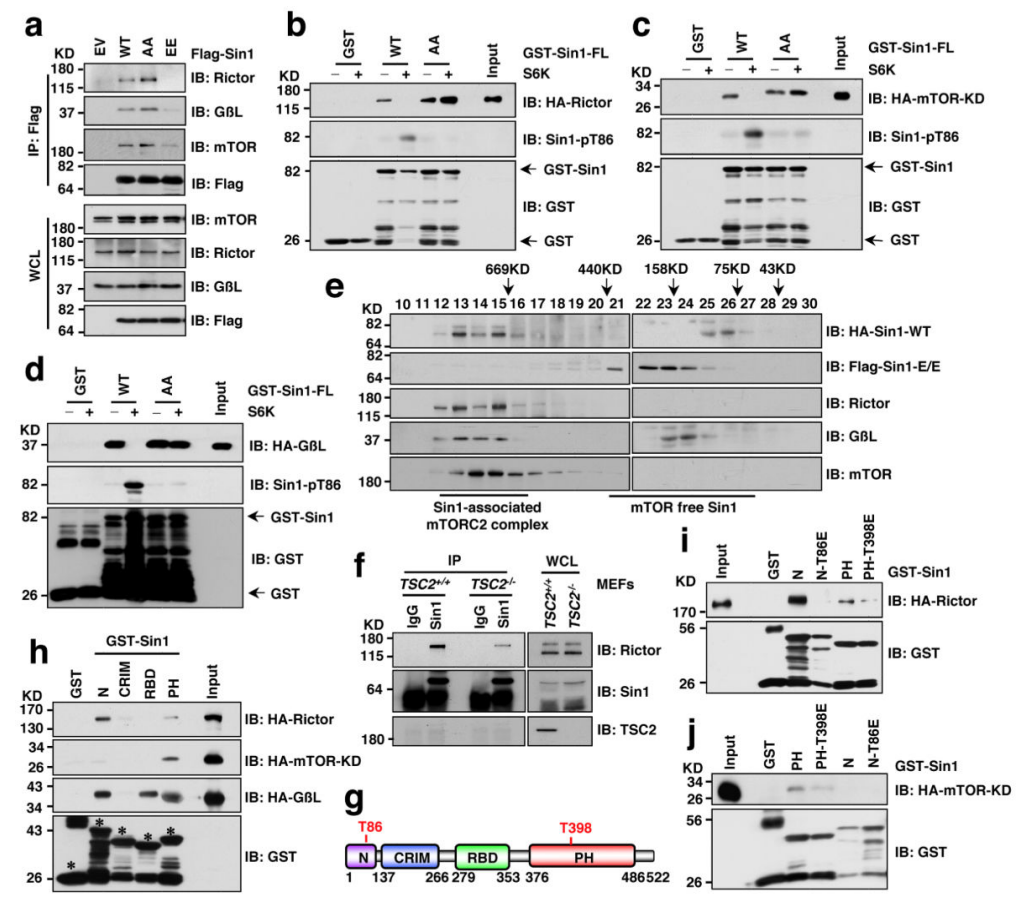

Figure 2. S6K-dependent phosphorylation of Sin1 dissociates Sin1 from the mTORC2 complex a. Immunoblot (IB) analysis of whole cell lysates (WCL) and Flag immunoprecipitates (IP) derived from 293T cells transfected with the indicated Flag-Sin 1 constructs (EV: empty vector control; WT: Sin1-WT; AA: Sin1-T86A/T398A; EE: Sin1-T86E/T398E).

b-d. GST pull down assays to demonstrate that S6K phosphorylation of GST-Sin1-WT-FL (full-length) but not GST-Sin1-T86A/T398A led to impaired interaction with Rictor (b), mTOR-kinase domain (KD) (c) or G $\beta \mathrm{L}(\mathbf{d})$. As indicated, GST-Sin1 proteins were phosphorylated by active recombinant S6K in vitro for 1 hour before using as a bait to pull down HA-Rictor (b), mTOR-kinase domain (KD) (c) or G $\beta L$ (d) expressed in 293T cells. e. Gel filtration experiments to illustrate that comparing with WT-Sin1, Sin1-EE lost interaction with the functional mTORC2 complex components in vivo. IB analysis of the indicated fractionations derived from the gel filtration experiment with HeLa cells cotransfected with HA-Sin1-WT and Flag-Sin1-EE constructs. Prior to running cell lysates, the molecular weight resolution of the column was first estimated by running native molecular weight markers (Thyroglobulin $\sim 669 \mathrm{KD}$, Ferritin $\sim 440 \mathrm{KD}$, Aldolase $\sim 158 \mathrm{KD}$, Conalbumin $\sim 75 \mathrm{KD}$ and Ovalbumin $\sim 44 \mathrm{KD}$ ) to determine their retention times on coomassie-stained SDS-PAGE protein gels.

f. Deletion of endogenous TSC2, which led to increased S6K kinase activity, resulted in a reduction of Rictor association with Sin1. IB analysis of WCL and anti-Sin1-IP derived from $\mathrm{TSC}^{+/+}$or $T S C 2^{-/-}$MEFs.

g. Schematic representation of the indicated domains of Sin 1 as well as the locations of the two Sin1 phosphorylation sites: T86 is in the N-terminal domain while T398 is located in the PH domain.

h. GST pull down assays to depict the Sin1 domains that interact with Rictor, mTOR-KD or $\mathrm{G} \beta \mathrm{L}$, respectively (* indicates the sizes of GST-Sin1 proteins). 
I-j. GST pull down assays to demonstrate that Sin1 T86E or T398E mutation led to reduced interaction with Rictor (i) or mTOR-KD (j), respectively. 


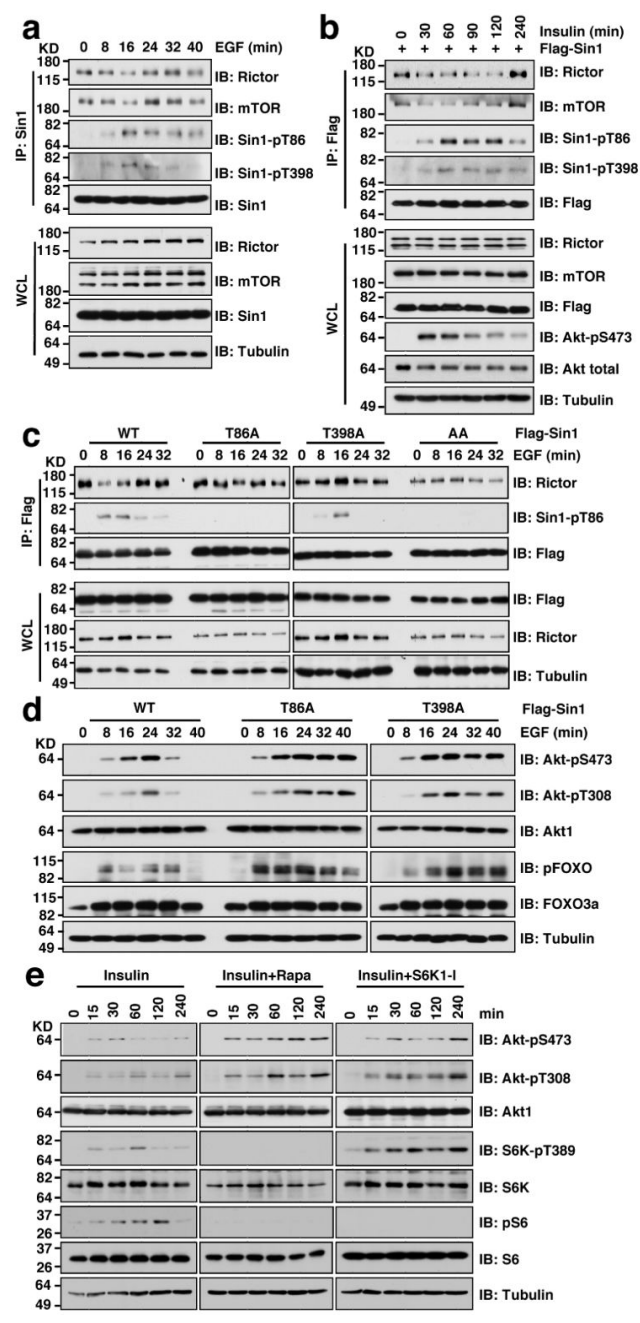

Figure 3. Sin1 phosphorylation induced by various stimuli impairs mTORC2 integrity a. Immunoblot (IB) analysis of whole cell lysates (WCL) and endogenous Sin1 immunoprecipitates (IP) derived from Sin1-WT MEFs that were serum-starved for 24 hours and then collected after EGF (100 ng/ml) stimulation for the indicated time periods.

b. IB analysis of WCL and Flag-IP derived from Flag-Sin1-transfected HeLa cells that were serum-starved for 24 hours and then collected after insulin stimulation for the indicated time periods.

c. Either Sin1-T86A or T398A mutation impaired the dynamic interaction between Sin1 and other essential mTORC2 components. IB analysis of WCL and Flag-IP derived from HeLa cells transfected with the indicated Flag-Sin 1 constructs that were serum starved for 12 hours and then treated with the EGF $(100 \mathrm{ng} / \mathrm{ml})$ for the indicated time periods before harvesting for IB analysis.

d. Either Sin1-T86A or T398A mutation led to sustained Akt activation upon EGF stimulation. IB analysis of WCL derived from HeLa cells transfected with the indicated Flag-Sin 1 constructs that were serum starved for 12 hours and then treated with the EGF $(100 \mathrm{ng} / \mathrm{ml})$ for the indicated time periods before harvesting for IB analysis. 
e. Rapamycin or S6K1-I treatment led to a relatively sustained Akt-pS473 upon insulin stimulation. IB analysis of WCL derived from HeLa cells serum starved for 12 hours and stimulated with $100 \mathrm{ng} / \mathrm{ml}$ insulin before harvesting at the indicated time points. Where indicated, $20 \mathrm{nM}$ rapamycin or $10 \mu \mathrm{M}$ S6K1-I was added. 


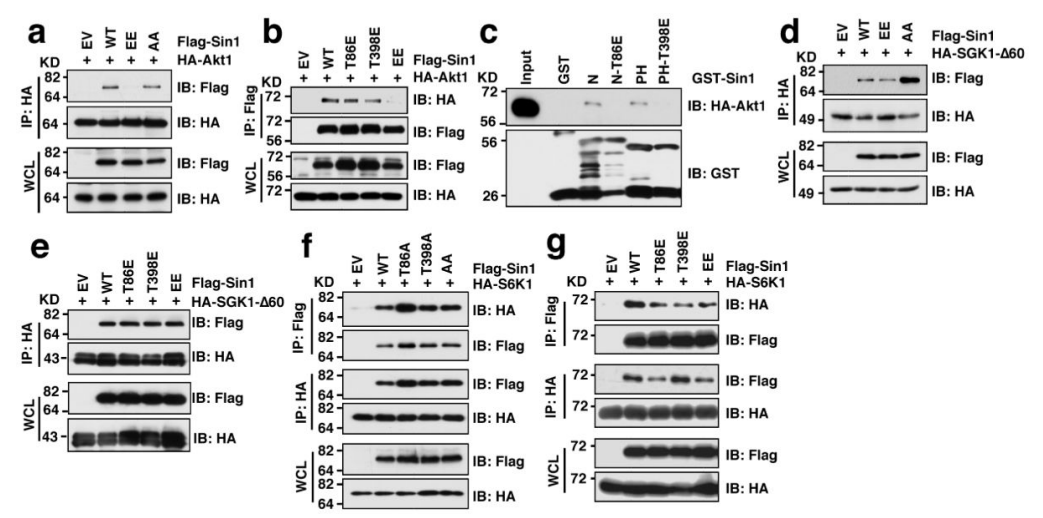

Figure 4. Sin1 phosphomimetic mutation is deficient in interacting with the mTORC2 substrate Akt1, but not SGK1

a-b. Immunoblot (IB) analysis of whole cell lysates (WCL) and HA (a) or Flag (b) immunoprecipitates (IP) derived from 293T cells that were transfected with the indicated Flag-Sin1 constructs with HA-Akt1.

c. Sin1-T86E or Sin1-T398E disrupts Sin1-N-terminus or Sin1-PH domain interaction with Akt1. Indicated GST-Sin1 proteins were used as a bait to pull down HA-Akt1 expressed in 293 T cells.

d-e. IB analysis of WCL and HA-IP derived from 293T cells that were transfected with the indicated Flag-Sin1 constructs with HA-SGK- $\Delta 60$.

f-g. IB analysis of WCL and HA-IP or Flag-IP derived from 293T cells that were transfected with the indicated Flag-Sin1 constructs with HA-S6K1. 

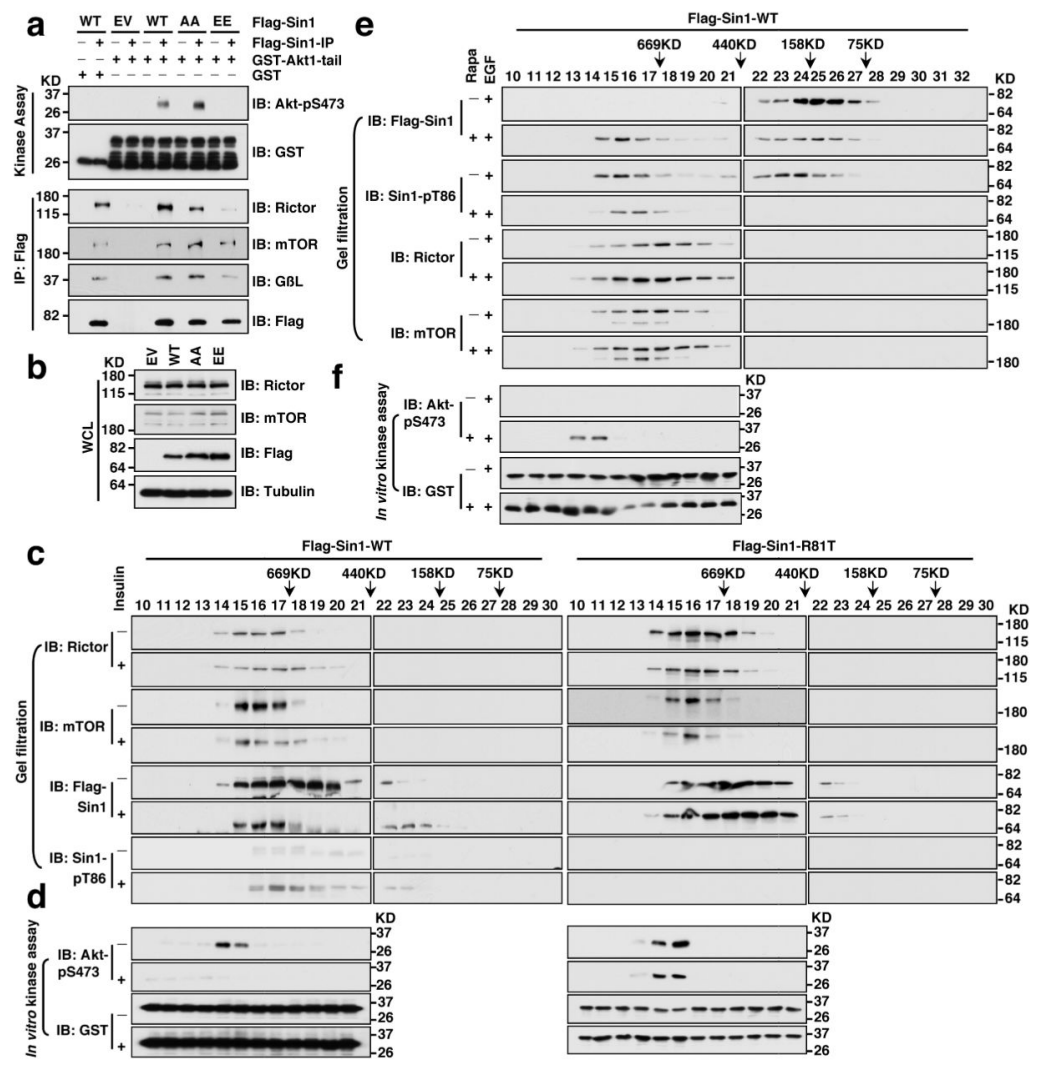

Figure 5. Sin1 phosphorylation suppresses mTORC2 kinase activity towards phosphorylating Akt in vitro

a-b. 293T cells were transfected with the indicated Flag-tagged Sin 1 constructs. 36 hours post-transfection, whole cell lysates (WCL) were collected and the mTORC2 complex was purified by Flag-immunoprecipitation (IP). The Flag-IPs were incubated in vitro with purified GST-Akt1 in the presence of ATP and the kinase reaction buffer. Thirty minutes later, the reaction was stopped by the addition of the loading buffer. Akt1 phosphorylation status was examined by immunoblot (IB) analysis.

c-d. Prolonged insulin treatment (45 $\mathrm{min}$ ) induces $\operatorname{Sin} 1$ phosphorylation, leading to dissociation of mTORC2 complex and abolished Akt activation. Flag-Sin1-WT or R81T mutant was transfected into HeLa cells and 48 hours later the transfected cells were harvested upon insulin (100 $\mathrm{nM})$ stimulation for $45 \mathrm{~min}$ after 12 hours of serum starvation in CHAPS buffer. The whole cell lysates were filtered and run through FPLC superdex 200 column. $500 \mu \mathrm{L}$ elute was collected for each fraction and 1/20 volume of each fraction was incubated with $2 \mu \mathrm{g}$ GST-Akt-tail (aa 408-480) at $30^{\circ} \mathrm{C}$ for $30 \mathrm{~min}$. Afterwards, the resulting samples were resolved on SDS-PAGE and subjected to IB analysis.

e-f. Rapamycin treatment restored Sin1 phosphorylation resulted from EGF treatment, leading to reassembly of mTORC2 complex and Akt activation. Flag-Sin1-WT was transfected into TSC2-depleted HeLa cells and 24 hours later, the transfected cells were treated with $20 \mathrm{nM}$ rapamycin for another 12 hours prior to EGF stimulation (100 nM) before harvested and analyzed as in (c-d). 

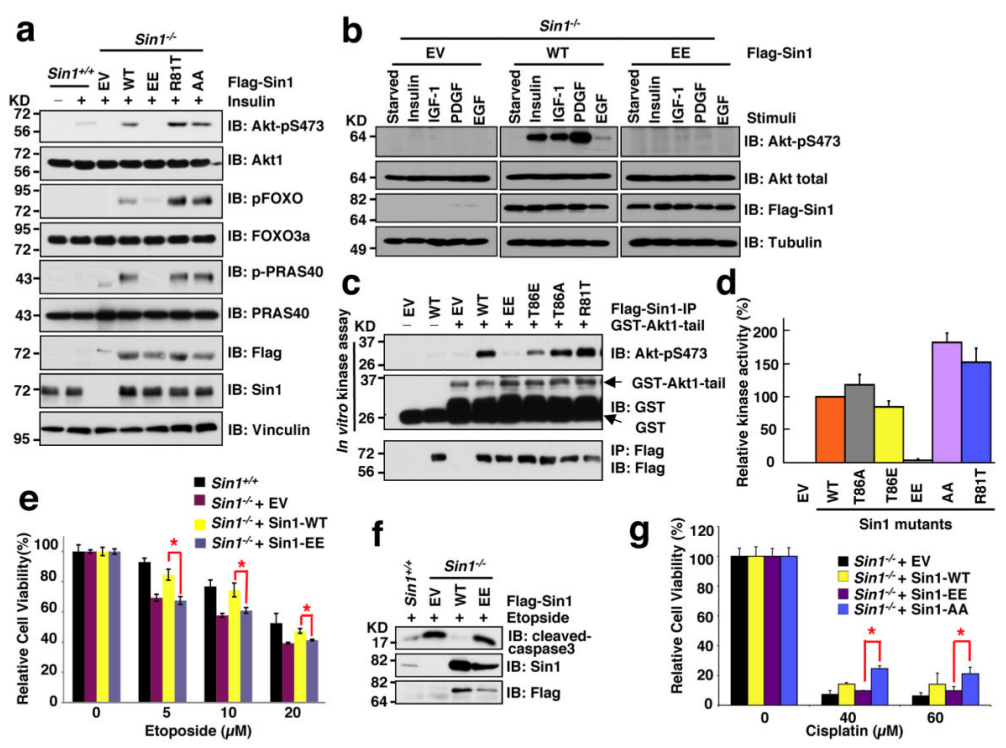

Figure 6. Sin1 phosphorylation attenuates mTORC2 kinase activity towards phosphorylating Akt in vivo

a. Sin1 $1^{-/-}$MEFs were transfected with the indicated Flag-Sin 1 constructs. 30 hours posttransfection, the resulting cells were serum-starved for 24 hours and then collected after stimulation with insulin for 30 minutes for immunoblot (IB) analysis.

b. Reconstitution of $\operatorname{Sin} 1^{-/-}$MEFs with WT-, but not EE-Sin1, could restore Akt-Ser473 phosphorylation under various stimulation conditions. Sin1 ${ }^{-/-}$MEFs were transfected with the indicated Flag-Sin 1 constructs and serum-starved for 24 hours before harvesting after treatment with indicated stimuli for IB analysis.

c. The indicated Flag-Sin1 constructs were transfected into Sin1 ${ }^{-/-}$MEFs and Flag immunoprecipitation (IP) was recovered as the kinase source to phosphorylate GST-Akt1tail (aa 408-480) in vitro.

d. The indicated Flag-Sin1 constructs were transfected into Sin1 ${ }^{-/-}$MEFs and endogenous Akt IP was performed as the kinase source to phosphorylate crosstide in vitro. Data was shown as mean \pm SD for $n=3$ independent experiments.

e-g. $\operatorname{Sin} 1^{-/-}$MEFs were transfected with the indicated Flag-Sin 1 constructs (with empty vector as a negative control). 24 hours post-transfection, the resulting cells were cultured in $10 \%$ FBS-containing medium with the indicated concentrations of etoposide (e) or cisplatin (f) for 48 hours before performing the cell viability assays (e,f) or IB analysis (g). Data was shown as mean $\pm \mathrm{SD}$ from $\mathrm{n}=3$ independent experiments. * indicates $p<0.05$ (Student's ttest). 


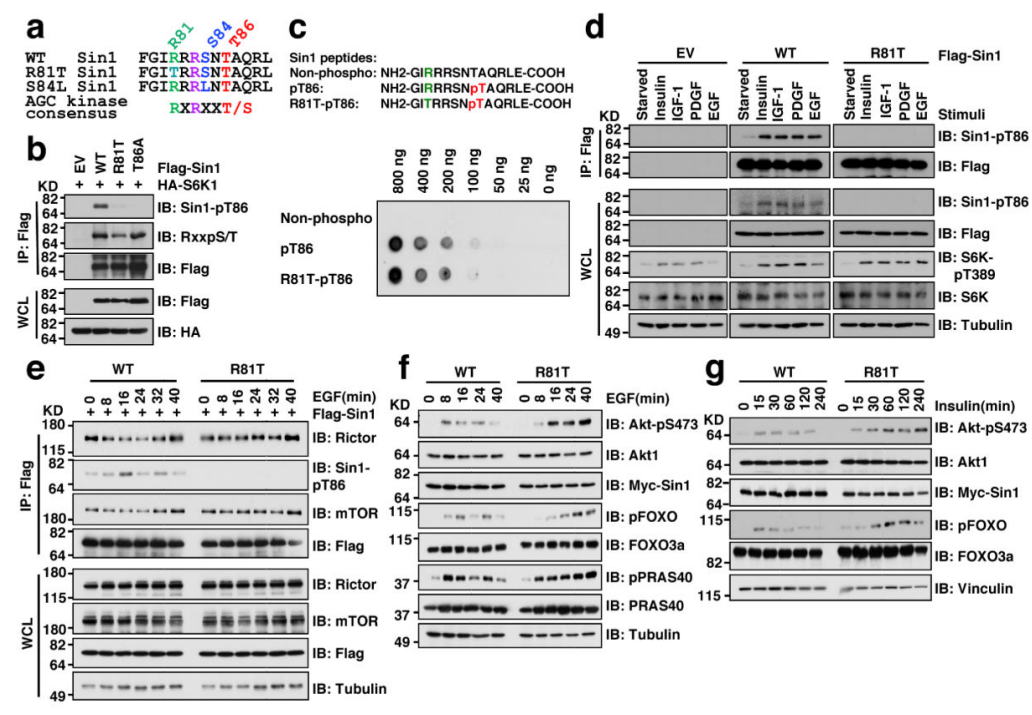

Figure 7. The pathological Sin1-R81T mutation led to attenuated Sin1-T86 phosphorylation and sustained Akt phosphorylation upon physiological stimulations

a. Schematic illustrations of the ovarian cancer patient-derived Sin1-R81T mutation and the skin cancer patient-derived S84L mutation.

b. Immunoblot (IB) analysis of whole cell lysate (WCL) and Flag-immunoprecipitates (IP) derived from $293 \mathrm{~T}$ cells transfected with the indicated Flag-Sin1 constructs and HA-S6K1. c. Sin1-R81T does not interfere with the Sin1-pT86 antibody to recognize Sin1-pT86.

Indicated Sin 1 synthetic peptides were dotted on nitrocellulose membrane for IB analysis. d. HeLa cells were transfected with the indicated Flag-Sin1 constructs and serum-starved for 24 hours and then collected after stimulation with the indicated stimuli for 30 minutes for IB analysis and Flag-IP.

e. $\operatorname{Sin} 1^{-/-}$MEFs were transfected with the indicated Flag-Sin 1 constructs and serum-starved overnight followed by IB analysis upon $100 \mathrm{ng} / \mathrm{ml}$ EGF stimulation for the indicated time points.

f-g. Sin1 depleted OVCAR5 cells stably expressing Sin1-WT or -R81T were serum starved overnight followed by IB analysis after treatment with $100 \mathrm{ng} / \mathrm{ml}$ EGF (f) or $100 \mathrm{nM}$ insulin (g) for the indicated time points. 

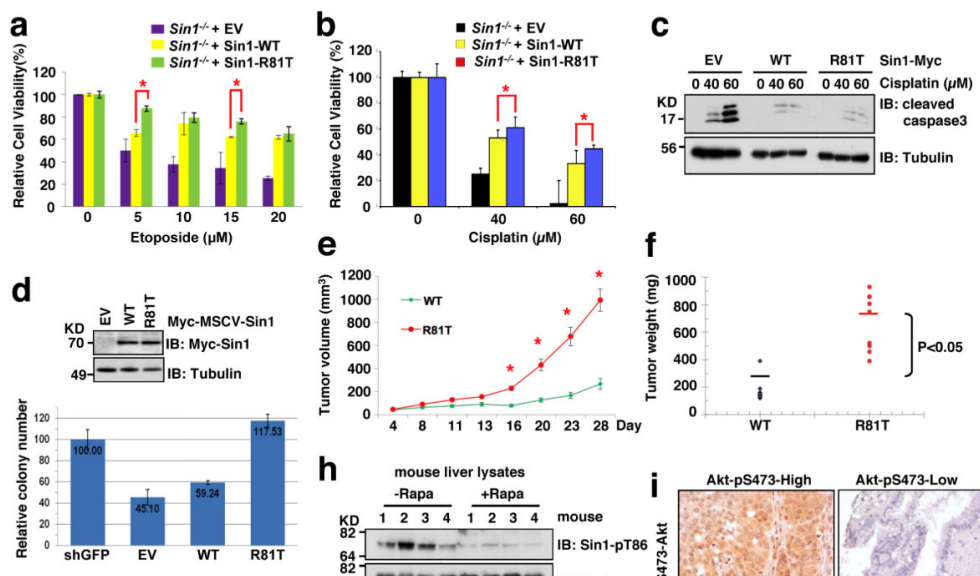

h
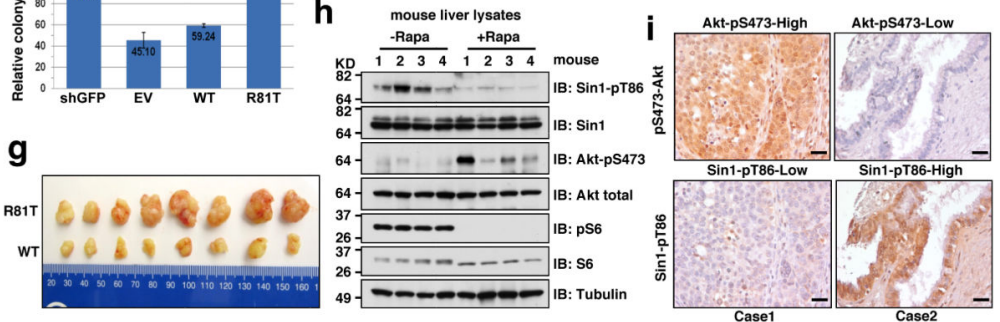

Figure 8. The pathological Sin1-R81T mutation displayed elevated oncogenic activity in part by bypassing Sin1 phosphorylation-mediated negative regulation of Akt-pS473

a-c. $\operatorname{Sin} 1^{-/-}$MEFs were transfected with the indicated Flag-Sin1 constructs and were treated with the indicated concentrations of etoposide (a) or cisplatin (b,c) for 48 hours before performing the cell viability assays (a,b) or immunoblot (IB) analysis (c). Data was shown as mean $\pm \mathrm{SD}$ from $\mathrm{n}=3$ independent experiments. * indicates $p<0.05$ (t-test).

d. Soft agar assays for Sin1-depleted OVCAR5 cells stably expressing EV, WT or R81T. Data was presented as mean $\pm \mathrm{SD}$ from $\mathrm{n}=3$ independent experiments.

e-f. Growth curves (e) and mass of the dissected tumors (f) from xenograft experiments with the indicated cells injected subcutaneously into $n=10$ mice for each cell line. The visible tumors were measured at the indicated days. Error bars, \pm SEM and * indicates $p<0.05$ (ttest).

g. Representative images of the dissected tumors presented in Figure 8e,f.

h. Eight-week-old mice were fasted overnight and then refed for 6 hours following a $30 \mathrm{~min}$ pretreatment with vehicle or rapamycin $(10 \mathrm{mg} / \mathrm{kg}) . \mathrm{N}=4$ mice per condition. Livers were dissected and liver lysates were subjected to IB analysis.

i. Four representative images of IHC with indicated Sin1 and Akt phosphorylation status out of 58 ovarian patient samples under 400x magnification. Scale bar represents $100 \mu \mathrm{m}$. 